\title{
Prognostic utility of ischemic response in functional imaging tests (SPECT or stress echocardiography) in low-risk unstable angina patients
}

Marcelo Trivi, Ricardo Ronderos, Alejandro Meretta, Diego Conde, Gustavo Avegliano

Division of Cardiology, Instituto Cardiovascular de Buenos Aires, Buenos Aires, Argentina

\begin{abstract}
Background: The aim of this study is to determine the ability of ischemic response in imaging stress tests (single-photon emission computed tomography [SPECT] or stress echocardiography [SE]) to predict events in low-risk unstable angina patients.

Methods: Three hundred and fifty-nine patients with unstable angina $(<24 \mathrm{~h})$, asymptomatic at admission, without ST-segment elevation or depression, normal troponins, and undergoing SPECT $(n=188)$ or $S E(n=171)$ during hospitalization (median $=1$ day) were included. A positive imaging test (IMAGING+) was defined as the presence of reversible perfusion defects or wall motion abnormalities in at least 2 contiguous segments. Multivariate models were constructed using these results and clinical variables to predict events at 6 months. Results: Ninety-nine (27\%) patients had IMAGING+, 72/188 (38\%) in SPECT and 27/17 (16\%) in SE $(p<0.0001)$. Events occurred in 84 (23\%) patients: 4 had myocardial infarction, 47 new hospitalizations due to angina and 33 coronary artery revascularizations. Independent predictors of coronary artery disease were: IMAGING + (OR: 6.4, 95\% CI: 3.4-11.8, $p<0.0001)$, history of coronary artery disease (OR: $2.5,95 \%$ CI: $1.2-5.2, p<0.02)$ and TIMI risk (OR: 1.5, 95\% CI: 1.1-2.2, $p<0.03$ ).

Conclusions: In low-risk unstable angina patients, an ischemic response in functional stress tests (SPECT or SE) was associated with adverse events and severe coronary artery disease. (Cardiol J 2015; 22, 2: 160-164)
\end{abstract}

Key words: unstable angina, functional test, prognostic

\section{Introduction}

The management of acute coronary syndromes (ACS) is a common challenge for a cardiologist. Nowadays, an invasive strategy characterized by coronary angiography followed by revascularization is clearly recommended in high-risk patients presenting with ST-segment or non-ST segment elevation ACS [1].

Yet, little attention has been paid to the management of patients initially stratified as having low risk, who represent a relatively high percentage of hospitalizations due to chest pain. Despite these patients have a favorable short-term outcome,

Address for correspondence: Diego Conde, MD, Cardiovascular Emergency Care Section, Instituto Cardiovascular de Buenos Aires, Blanco Encalada 1543, Postal Code: 1428, Buenos Aires, Argentina, tel/fax: (11) 4787-7500, ext. 3133, e-mail: drconde@hotmail.com

Received: 31.05.2014 Accepted: 13.06.2014 
approximately $15 \%$ will suffer a new event within the first year, particularly new hospitalization and revascularization $[2,3]$.

Guidelines recommend hospitalized low-risk patients with negative troponins, absence of electrocardiographic abnormalities and who remain without symptoms to undergo an exercise stress test for further risk stratification. Imaging tests provide higher diagnostic yield, but are recommended in special cases [1, 4].

In our environment, imaging tests, particularly sestamibi cardiac scan (single-photon emission computed tomography - SPECT) or stress echocardiography (SE), are frequently used to evaluate patients with ACS once they become stable. The value of both tests in chronic patients is unquestionable; yet, their usefulness in patients with ACS, which constitutes a completely different scenario, has not been adequately explored [5-10].

The aim of the present study is to determine if the ischemic response in imaging tests is related with an unfavorable outcome in patients hospitalized with low-risk unstable angina without significant predictors of risk.

\section{Methods}

All the patients hospitalized due to definite or probable unstable angina between 2008 and 2010, and who underwent imaging tests during the hospitalization at Instituto Cardiovascular de Buenos Aires were considered for the study. Then, patients with the following criteria were selected: (1) Angina within $24 \mathrm{~h}$ before admission; (2) Absence of symptoms during hospitalization; (3) Absence of electrocardiographic abnormalities suggestive of acute ischemia; (4) Troponin $\mathrm{T}<0.01 \mathrm{ng} / \mathrm{mL}$; (5) Absence of significant valvular heart disease of cardiomyopathies.

A total of 359 patients with these criteria were enrolled during 2 years and were followed-up during 6 months. These patients were evaluated with SPECT $(\mathrm{n}=188)$ or SE $(\mathrm{n}=171)$ during hospitalization (median $=1$ day) according to each patient's characteristics. Most patients (324; 90\%) underwent exercise stress testing, and pharmacological stress testing was used in the rest. The information about the demographic characteristics, clinical data and outcomes were retrieved from the medical records. For each patient, a Thrombolysis in Myocardial Infarction (TIMI) risk score was assigned at admission [11].

\section{Protocols of imaging stress tests}

Imaging stress tests, either with exercise or with pharmacological stress, were performed within $48 \mathrm{~h}$ after admission (normally in the first $24 \mathrm{~h}$ ). The type of stress was selected according to the availability of the test, the preference of the treating physician and the patient's ability to exercise.

SPECT: After an intravenous line was placed, the patient underwent an exercise stress test in bicycle ergometer or treadmill, or received intravenous dipyridamole, $40 \mathrm{mg}$ (as pharmacological stress) followed by intravenous aminophylline. Tc 99m-labeled sestamibi was injected at peak stress, and images were acquired after eating a fatty meal. Rest images were acquired after a second infusion, during the same day. SPECT was considered positive (SPECT + ) in the presence of reversible perfusion defects (improved defect at rest) in at least 2 contiguous segments.

SE: The test was performed following the recommendations of the American Society of Echocardiography [12], using a Philips Envisor HD ultrasound system with digital images and integrated acquisition protocols, using second harmonic imaging. Exercise was performed in a supine bicycle bed and images were acquired at rest, during exercise and after exercise. For pharmacological stress, dobutamine was administered by intravenous infusion at incremental doses plus atropine to achieve $85 \%$ of the theoretical maximum heart rate.

Stress echocardiography was considered positive $(\mathrm{SE}+)$ when new or worsening wall motion abnormalities developed in at least 2 contiguous segments.

Finally, positive imaging test (IMAGING+) was defined as SPECT + or SE+.

Coronary angiography: Only 63 (18\%) patients with inducible ischemia or new hospitalization due to angina and suspected coronary artery disease $(\mathrm{CAD})$ underwent coronary angiography, as the guidelines consider that performing this method to low-risk patients without inducible ischemia or symptoms is not ethical. Severe CAD was defined as stenosis $\geq 70 \%$ measured by quantitative angiography in at least one coronary artery.

Follow-up: Only patients who had been followed up during 6 months by review of the electronic medical record or by telephone were included.

\section{Statistical analysis}

Continuous variables were expressed as mean and median, and analyzed using the $t$ test or the Wil- 
Table 1. Differences between patients with positive and negative tests.

\begin{tabular}{|c|c|c|c|}
\hline Variable & $\begin{array}{l}\text { IMAGING (+) } \\
N=99(28 \%)\end{array}$ & $\begin{array}{l}\text { IMAGING (-) } \\
N=260(73 \%)\end{array}$ & $\mathbf{P}$ \\
\hline Age & $63 \pm 10$ & $56 \pm 11$ & $<0.01$ \\
\hline Female & $14(14 \%)$ & $87(33 \%)$ & $<0.05$ \\
\hline Diabetes mellitus & $23(23 \%)$ & $33(13 \%)$ & $<0.05$ \\
\hline Previous myocardial infarction & $42(42 \%)$ & $48(20 \%)$ & $<0.01$ \\
\hline TIMI risk score & $2.64 \pm 1.04$ & $1.78 \pm 1.23$ & $<0.01$ \\
\hline History of coronary artery disease & $65(65 \%)$ & $65(25 \%)$ & $<0.01$ \\
\hline
\end{tabular}

coxon signed-rank test according to the distribution. Discrete variables were expressed as percentages and analyzed by the $\chi^{2}$ test. All the variables with a $\mathrm{p}$ value $<0.10$ at the univariate analysis were included in the multivariate logistic regression analysis to detect severe coronary stenosis. Multivariate models were constructed, considering the total group (IMAGING). All the calculations were performed using the StatsDirect 1.7.3 software.

\section{Results}

Correlation between the clinical data, demographics and imaging tests

An ischemic response (IMAGING+) was seen in 99 (28\%) patients: $38 \%$ with SPECT and $16 \%$ with SE $(\mathrm{p}<0.01)$. The characteristics of the patients with positive and negative tests are compared in Table 1. Patients with ischemic tests were older, diabetics, men, had a history of infarction or CAD and a higher TIMI risk score.

Correlation between the results of imaging tests and invasive coronary angiography.

Sixty-three (18\%) patients underwent coronary angiography, 50/99 with ischemic response (50\%) and 13/260 (5\%) without ischemic response $(\mathrm{p}<0.01)$. Severe CAD was detected in $56 / 63$ (89\%) patients. Patients with ischemic response presented more evidence of documented CAD than the rest (44/99 and 12/269, $\mathrm{p}<0.001)$. Severe CAD was present in $27 / 31$ (87\%) patients with ischemic response by SPECT and in 17/19 (89\%) patients with ischemic response by SE ( $\mathrm{p}=\mathrm{NS})$.

Table 2 shows the independent predictors of severe $\mathrm{CAD}$ after multivariate logistic analysis. An image test with ischemic response was the main independent predictor, increasing the risk of having severe CAD by 13 times, which was above historical variables as history of $\mathrm{CAD}$ and TIMI risk score.
Table 2. Significant predictors of severe coronary artery disease (CAD) at multivariate analysis ( $p<0.03)$.

\begin{tabular}{lcc}
\hline Variable & Odds ratio & $95 \% \mathrm{Cl}$ \\
\hline Ischemic response & 13.1 & $5.9-30.0$ \\
History of CAD & 3.7 & $1.6-8.7$ \\
TIMI risk score $>2$ & 2.7 & $1.1-6.4$ \\
\hline
\end{tabular}

$\mathrm{Cl}$ - confidence interval

Clinical follow-up and cardiovascular events Events were detected in $23 \%$ of the 359 patients, and included 4 acute myocardial infarctions, 47 new hospitalizations due to angina and 33 coronary artery revascularizations (27 percutaneous coronary interventions and 6 coronary artery bypass graft surgeries). The results of the tests and the events are shown in Table 3.

\section{Outcome predictors}

The ischemic response in the imaging test was the most important independent predictor, increasing the risk of events by 6.4 times. The other significant clinical variables were history of CAD and TIMI risk score, increasing the risk by 2.5 and 1.5 times, respectively. Even after excluding revascularization, an imaging test with ischemic response was a predictor of events. Table 4 shows significant independent predictors identified by logistic regression.

\section{Discussion}

The management of patients with ACS has become standardized. Most recommendations agree in the necessity of interventional therapy in high-risk patients. In the same sense, low-risk patients should not be intervened as they have better outcomes and revascularization does not offer greater advantages in these patients. How- 
Table 3. Ischemic response and events at 6 months.

\begin{tabular}{lcccc}
\hline Variable & $\begin{array}{c}\text { Total } \\
(\mathbf{n}=359)\end{array}$ & $\begin{array}{c}\text { SPECT group } \\
(\mathbf{n}=188)\end{array}$ & $\begin{array}{c}\text { SE group } \\
(\mathbf{n}=171)\end{array}$ & P \\
\hline Ischemic response & $99(27 \%)$ & $72(38 \%)$ & $27(16 \%)$ & $<0.0001$ \\
New hospitalization & $42(12 \%)$ & $25(13 \%)$ & $17(10 \%)$ & NS \\
Acute myocardial infarction & $4(1 \%)$ & $4(2 \%)$ & $0(0 \%)$ & NS \\
Revascularization & $38(11 \%)$ & $27(14 \%)$ & $11(5 \%)$ & $<0.03$ \\
Total events & $84(23 \%)$ & $54(29 \%)$ & $30(18 \%)$ & $<0.02$ \\
Events excluding revascularization & $46(13 \%)$ & $27(14 \%)$ & $19(11 \%)$ & NS \\
\hline
\end{tabular}

Table 4. Significant predictors of events in multivariate analysis.

\begin{tabular}{|c|c|c|}
\hline Variable & $\begin{array}{l}\text { Total events } \\
\text { OR ( } 95 \% \mathrm{CI})\end{array}$ & $\mathbf{P}$ \\
\hline IMAGING $(+)(n=359)$ & $6.4(3.4-11.8)$ & $<0.01$ \\
\hline $\begin{array}{l}\text { History of coronary } \\
\text { artery disease }\end{array}$ & $2.5(1.2-5.2)$ & $<0.002$ \\
\hline TIMI risk score & $1.5(1.1-2.2)$ & $<0.03$ \\
\hline $\begin{array}{l}\text { SPECT positive } \\
(\mathrm{n}=188)\end{array}$ & $3.9(1.7-8.5)$ & $<0.02$ \\
\hline $\begin{array}{l}\text { Stress echocardiography } \\
\text { positive }(n=171)\end{array}$ & $9.2(2.52-33.8)$ & $<0.001$ \\
\hline
\end{tabular}

ever, among the patients discharged from hospital with diagnosis of low-risk unstable angina, 1 or 2 out of 10 will require new hospitalization for new symptoms, unusually due to myocardial infarction, or will require new revascularization (either by percutaneous intervention or surgery) within 6 months after discharge.

Detecting the patients who will develop events among the low-risk group has always been complicated. However, a high percentage of events occur in these patients, who are very frequent in clinical practice. These patients should be differentiated from those evaluated in the emergency room with doubtful presentations, constituting a group with lower risk requiring hospitalization in a very small percentage of cases.

There are few publications of low-risk unstable angina patients undergoing any imaging stress test, including conventional exercise stress test, SPECT or SE [13-17]. Despite the prognostic significance of these tests is different in acute patients, the results are interpreted using the risk criteria for stable angina.
In our environment, SPECT is frequently used for risk stratification and prognostic evaluation. The decision to use SPECT or SE depends, in most cases (as it happened in this study) on the availability of each test, trying to avoid hospital delays.

The results of our study suggest that the presence of an ischemic response in imaging stress tests predicts the development of events in the shortterm. One of 4 patients will exhibit an ischemic response (defined as transient perfusion defects or wall motion abnormalities) and in these patients the risk of events is 6 times higher and statistically significant, with a narrow confidence interval.

This diagnostic yield of imaging stress tests in the group classified by the guidelines as low-risk unstable angina patients is greater than the one of the other predictors analyzed in this sample: history of CAD and TIMI risk score widely used in the coronary care units for prognostic stratification.

The decision between using SPECT or SE was not the aim of this study, and the question of which test is better cannot be answered with the information obtained. Both tests were independent predictors of events, including revascularization.

New hospitalizations and revascularization are considered soft cardiac events, and revascularization cannot be independent of the result of the functional test. However, hard cardiac events, as mortality or myocardial infarction, are difficult to detect in a low-risk population [18]. In addition, keeping the result of the test secret would have been the only way to make revascularization independent of the result of the tests, but this can only be achieved with a prospective and blind design.

For this reason, we also analyzed the predictive value of the tests to detect $\mathrm{CAD}$ in diagnostic coronary angiography. Also in this case the ischemic response was a predictor of severe coronary artery stenosis. Yet, the percentage of coronary 
angiographies was low and conditioned by the results of the stress tests.

\section{Conclusions}

In low-risk unstable angina patients, an ischemic response in functional stress tests (SPECT or SE) is more precise than clinical parameters as history of CAD and TIMI risk score to predict events at 6 months, particularly new hospitalization, revascularization and severe coronary artery stenosis. These tests seem to be useful to guide a medical or an invasive strategy in this group of patients.

\section{Conflict of interest: None declared}

\section{References}

1. Hamm CW, Bassand JP, Agewall S et al. ESC Guidelines for the management of acute coronary syndromes in patients presenting without persistent ST-segment elevation: The Task Force for the management of acute coronary syndromes (ACS) in patients presenting without persistent ST-segment elevation of the European Society of Cardiology (ESC). Eur Heart J, 2011; 32: 2999-3054.

2. Nørgaard BL, Andersen K, Thygesen K et al. Long term risk stratification of patients with acute coronary syndromes: Characteristics of troponin $\mathrm{T}$ testing and continuous ST segment monitoring. Heart, 2004; 90: 739-744.

3. Cannon CP, Weintraub WS, Demopoulos LA et al. TACTICS (Treat Angina with Aggrastat and Determine Cost of Therapy with an Invasive or Conservative Strategy): Thrombolysis in Myocardial Infarction 18 Investigators. Comparison of early invasive and conservative strategies in patients with unstable coronary syndromes treated with the glycoprotein IIb/IIIa inhibitor tirofiban. N Engl J Med, 2001; 344: 1879-1887.

4. Anderson JL, Adams CD, Antman EM et al. ACC/AHA 2007 guidelines for the management of patients with unstable angina/ /non-ST-elevation myocardial infarction: A report of the American College of Cardiology/American Heart Association Task Force on Practice Guidelines (Writing Committee to Revise the 2002 Guidelines for the Management of Patients With Unstable Angina/Non-ST-Elevation Myocardial Infarction) developed in collaboration with the American College of Emergency Physicians, the Society for Cardiovascular Angiography and Interventions, and the Society of Thoracic Surgeons endorsed by the American Association of Cardiovascular and Pulmonary Rehabilitation and the Society for Academic Emergency Medicine. J Am Coll Cardiol, 2007; 50: e1-e157.

5. Gaibazzi N, Squeri A, Reverberi C et al. Contrast stress-echocardiography predicts cardiac events in patients with suspected acute coronary syndrome but nondiagnostic electrocardiogram and normal 12-hour troponin. J Am Soc Echocardiogr, 2011; 24: 1333-1341.

6. Geleijnse ML, Elhendy A, Kasprzak JD et al. Safety and prognostic value of early dobutamine-atropine stress echocardiography in patients with spontaneous chest pain and a non-diagnostic electrocardiogram. Eur Heart J, 2000; 21: 397-406.

7. Bholasingh R, Cornel JH, Kamp O et al.. Prognostic value of predischarge dobutamine stress echocardiography in chest pain patients with a negative cardiac troponin T. J Am Coll Cardiol, 2003; 41: 596-602.

8. Petix NR, Sestini S, Coppola A et al. Prognostic value of combined perfusion and function by stress technetium-99m sestamibi gated SPECT myocardial perfusion imaging in patients with suspected or known coronary artery disease. Am J Cardiol, 2005; 95: 1351-1357.

9. Wang CH, Cherng WJ, Hung MJ, Kuo LT. Short- and long-term prognostic value of cardiac troponin I and dobutamine echocardiography in patients with stabilized acute coronary syndromes. Int J Cardiol, 2001; 80: 193-200.

10. Dakik HA, Hwang WS, Jafar A, Kimball K, Verani MS, Mahmarian JJ. Prognostic value of quantitative stress myocardial perfusion imaging in unstable angina patients with negative cardiac enzymes and no new ischemic ECG changes. J Nucl Cardiol, 2005; 12: 32-36.

11. Antman EM, Cohen M, Bernink PJ et al. The TIMI risk score for unstable angina/non-ST elevation MI: A method for prognostication and therapeutic decision making. JAMA, 2000; 284: 835-842.

12. 2011 Appropriate Use Criteria for Echocardiography. A Report of the American College of Cardiology Foundation Appropriate Use Criteria Task Force, American Society of Echocardiography,American Heart Association, American Society of Nuclear Cardiology, Heart Failure Society of America, Heart Rhythm Society, Society for Cardiovascular Angiography and Interventions, Society of Critical Care Medicine, Society of Cardiovascular Computed Tomography, Society for Cardiovascular Magnetic Resonance American College of Chest Physicians. J Am Soc Echocardiogr, 2011; 24: 229-267.

13. Markman Filho B, Almeida MC, Markman M et al. Stratifying the risk in unstable angina with dobutamine stress echocardiography. Arq Bras Cardiol, 2006; 87: 294-299.

14. de Azevedo JC, Félix RC, Corrêa PL et al. Medium term prognostic value of stress myocardial perfusion scintigraphy in a chest pain unit. Arq Bras Cardiol, 2007; 88: 602-610.

15. Gaibazzi N, Reverberi C, Badano L. Usefulness of contrast stressechocardiography or exercise-electrocardiography to predict long-term acute coronary syndromes in patients presenting with chest pain without electrocardiographic abnormalities or 12-hour troponin elevation. Am J Cardiol, 2011; 107: 161-167.

16. Alvarez TamargoJA, Martin-Ambrosio ES, Tarin ER, FernandezMM, De la Tassa CM. Significance of the treadmill scores and highrisk criteria for exercise testing in non-high-risk patients with unstable angina and an intermediate Duke treadmill score. Acta Cardiol, 2008; 63: 557-564.

17. Dorbala S, Giugliano RP, Logsetty G et al. Prognostic value of SPECT myocardial perfusion imaging in patients with elevated cardiac troponin I levels and atypical clinical presentation. J Nucl Cardiol, 2007; 14: 53-58.

18. Kelly AM. What is the incidence of major adverse cardiac events in emergency department chest pain patients with a normal ECG, Thrombolysis in Myocardial Infarction score of zero and initial troponin < =99th centile: an observational study? Emerg Med J, 2013; 30: 15-18. 